\title{
Raconter des histoires pour développer des compétences transversales en FLE
}

\section{Tell stories to develop transversal competencies in FLE}

\author{
Marzena Blachowska-Szmigiel \\ Université Adam Mickiewicz de Poznań \\ majek@amu.edu.pl
}

\begin{abstract}
The purpose of this paper is to seek to highlight the usefulness of storytelling skills for the development of transversal competencies in the context of FLE teaching / learning in Romance philology at Adam Mickiewicz University. As a first step we define the importance of transversal competencies in developing the general communicative competence of the student as a participant in social life, then we go on to describe narrative competence as transversal competence and to show its role in managing the 'SOI' integrity, finally, we concentrate on developing narrative competence on Roman philology at Adam Mickiewicz University.
\end{abstract}

Keywords: transversal competencies, narrative competence, 'SOI' integrity, FLE teaching / learning

\section{INTRODUCTION}

Dans la présente étude, nous cherchons à mettre en valeur le caractère transversal de la compétence narrative. Les enjeux de la compétence narrative sont multiples et son rôle dans la communication quotidienne reste incontournable. Comme le remarque A. Dizerbo : "Grâce au récit, l'auteur-narrateur inscrit son existence dans un environnement social et culturel, dans la continuité de son histoire, dans une relation aux autres. L'apprentissage de cette compétence narrative représente donc un enjeu primordial » (Dizerbo, 2014, p. 5). L'activité narrative permet au sujet communicant (à l'apprenant-acteur social) de situer son vécu dans un cadre spatio-temporel et de construire/reconstruire son identité à travers son 
histoire ainsi que de comprendre/interpréter les expériences de l'autre. Elle soustend les prises de contact, l'établissement des relations, l'exécution des rôles sociaux. Ce qui nous intéresse c'est l'autonarration en tant que déclencheur d'une réflexion approfondie sur soi-même et en tant qu'outil de gestion de l'intégrité des «SOI». Nous visons à utiliser l'autonarration pour faciliter aux étudiants de la philologie romane une réflexion sur «qui je suis» (le SOI réel) et sur « qui je voudrais être » (le SOI idéal). Une telle prise de conscience soutient la détermination des objectifs personnels/professionnels à court terme (un mois, six mois, un an), à long terme (entre cinq et dix ans), les objectifs de vie, permettant ainsi d'apprivoiser ses rêves, de les rendre plus tangibles. Nous cherchons d'abord à montrer comment le Cadre européen commun de Référence pour les langues (2001) aborde la question de la transversalité des compétences. Nous nous concentrons ensuite sur la transversalité de la compétence narrative et sur le rôle de la compétence autonarrative dans la gestion de l'intégrité de "SOI». Ceci nous conduit finalement aux caractéristiques du développement de la compétence narrative en philologie romane lesquelles nous complétons par l'exemple d'une mise en pratique de l'utilisation de l'autonarration en tant que déclencheur d'une réflexion sur le « SOI » réel et le SOI idéal.

\section{LA TRANSVERSALITÉ DES COMPÉTENCES DU SUJET COMMUNICANT - ACTEUR SOCIAL DANS LA PERSPECTIVE DU CADRE EUROPÉEN COMMUN DE RÉFÉRENCE POUR LES LANGUES}

Privilégiant une perspective actionnelle, le CECRL considère l'apprenant d'une langue (tout aussi bien que l'usager) comme « un acteur social» (CECRL, 2001, p. 9), «un utilisateur compétent et expérimenté » (CECRL, 2001, p. 5), qui a «à accomplir des tâches (qui ne sont pas seulement langagières) dans des circonstances et un environnement donnés à l'intérieur d'un domaine d'action donné » (CECRL, 2001, p. 15). Par les compétences, le CECRL comprend «l'ensemble des connaissances, des habiletés et des dispositions qui permettent d'agir » (CECRL, 2001, p. 15) et les envisage dans une perspective plurilingue et pluriculturelle ce qui demande leur accommodation au défi de maîtriser plusieurs langues et de gérer les contacts avec plusieurs cultures.

Le développement de la compétence plurilingue et pluriculturelle implique une intensification de l'enseignement/apprentissage des langues et un prolongement de l'apprentissage des langues tout au long de la vie (CECRL, 2001, p. 11). Par ailleurs, ces postulats trouvent une justification dans la recherche sur l'impact du bilinguisme sur le cerveau. Il s'avère que les bilingues font face à la vie sociale plus efficacement que les monolingues grâce aux avantages suivants : la flexibilité mentale, l'habileté à résoudre des problèmes, les capacités métalinguistiques (une capacité 
à « lire entre les lignes »), la capacité à apprendre par l'utilisation d'une information donnée et par la création des solutions nouvelles à partir des informations acquises, la capacité à créer des relations interpersonnelles, le vieillissent mental de deux à quatre ans plus tard (Bertelle, 2011, p. 243).

De plus, la perspective plurilingue et pluriculturelle conduit à une mise en valeur de la compétence partielle (CECRL, 2001, p. 128) et engage une réflexion sur les rapports et les frontières entre le partiel et le transversal : "Toute connaissance partielle est aussi moins partielle qu'il n'y paraît. Par exemple, pour atteindre l'objectif limité de la capacité à comprendre, dans une langue étrangère donnée, des textes spécialisés de telle ou telle discipline que l'on maîtrise, il faut acquérir des connaissances et des habiletés utilisables pour d'autres buts. Toutefois, ces retombées concernent plus l'apprenant que le planificateur» (CECRL, 2001, p. 129). Admettant que les compétences sont constamment actualisées à partir de l'expérience du sujet communicant, le CECRL les approche d'une façon constructiviste. Les frontières entre «le partiel » et «le transversal» restent floues et dépendent du niveau de la compétence langagière globale du sujet apprenant-acteur social, à savoir du niveau de sa compétence à communiquer langagièrement et de ses compétences générales individuelles. Le développement de la compétence à communiquer langagièrement est affecté par les compétences générales individuelles qui s'enrichissent elles-mêmes de par l'apprentissage d'une autre langue.

La compétence à communiquer langagièrement est constituée des compétences linguistique, sociolinguistique, pragmatique et découpée en capacités langagières de réception (écouter, lire), de production (parler, écrire, individuellement ou en interaction) et de médiation (traduire/interpréter). Les compétences générales individuelles ont le caractère transversale car « tout type de tâche requiert que soit activé un ensemble de compétences générales appropriées » (CECRL, 2001, p. 121) ${ }^{1}$.

Les compétences générales individuelles embrassent :

- la connaissance du monde qui couvrent les savoirs empiriques résultant de l'expérience sociale et les savoirs académiques relevant de l'apprentissage formel,

- les habiletés et les savoir-faire résultant du savoir procédural,

- les savoir-être c'est-à-dire des dispositions individuelles, des dispositifs d'attitudes et des traits de personnalité (qui influent sur le rôle du sujet apprenant dans sa réalisation de l'activité), des savoir-apprendre (qui affectent des savoir-être, des savoirs et des savoir-faire et puisent dans des compétences de différents types).

\footnotetext{
${ }^{1}$ Elles sont de plus en plus recherchées sur le marché du travail : « la différence entre deux candidatures a priori égales se fait désormais sur le savoir-être : plus le candidat est adaptable, optimiste, créatif ou doté d'un esprit d'équipe, plus il séduira le recruteur » (www.lemonde.fr/emploi/article/ 2016/10/12/en-entreprise-la-personnalite-des-diplomes-devientprimordiale_5012299_1698637.html).
} 
Le CECRL ne donne pas une réponse univoque quant à la façon d'approcher les connaissances générales individuelles pendant l'enseignement/apprentissage. En postulant « de favoriser le développement harmonieux de la personnalité de l'apprenant et de son identité »(CECRL, 2001, p. 9) il se contente de constater qu' "il revient aux enseignants et aux apprenants eux-mêmes de construire une personnalité saine et équilibrée à partir des éléments variés qui la composeront » (CECRL, 2001, p. 9). En soulignant l'impact enrichissant de l'expérience pluriculturelle et plurilingue sur les traits de personnalité et l'identité de l'apprenant, en évoquant même l'idée «du développement d'une personnalité interculturelle » (CECRL, 2001, p. 85), le CECRL ne les approfondit pas.

La progression de la compétence langagière globale est représentée en échelle de trois niveaux : d'un utilisateur élémentaire (A), d'un utilisateur indépendant (B) et d'un utilisateur expérimenté $(C)$. Ceux-ci sont encore subdivisés en six niveaux dont chacun est accompagné d'un descripteur de la compétence langagière globale respective. De cette gradation relèvent trois bases des compétences transversales dont le sujet communicant a besoin afin de réaliser des tâches prévues pour le niveau donné, par exemple pour un utilisateur élémentaire du niveau A1 la capacité à formuler des phrases simples de type « sujet-verbe-objet » relève d'une compétence transversale mais elle ne l'est plus pour un utilisateur indépendant du niveau B1.

L'approche dynamique et constructiviste des compétences adoptée par le CECRL amène à constater que plus le sujet communicant évolue vers le niveau d'un utilisateur expérimenté et plus il accroît ses compétences générales, plus il est censé appréhender les compétences de réception, de production, de médiation en langue seconde (en d'autres langues) d'une façon transversale. En d'autres termes, il est capable de les traiter comme des outils pour négocier, résoudre des problèmes, inventer des idées, établir des relations, gérer l'intégrité de son identité, enfin gérer le développement de son potentiel. Pour reprendre l'exemple donné par J.C. Coulet dans la perspective ontogénétique : « on peut considérer que, pour un bébé, sucer est une compétence transversale, dans la mesure où elle couvre une classe de tâches extrêmement étendue mais, bien évidemment ce n'est déjà plus le cas pour un jeune enfant et, encore moins pour un adulte ! De la même manière mais à l'inverse, lire est une compétence transversale pour un adulte cultivé car elle couvre une classe de tâches extrêmement large mais reste longtemps une compétence très spécifique pour un enfant de cours préparatoire » (Coulet, 2016, p. 25).

C'est ainsi, compte tenu des stades du développement des schémas cognitifs vers des structures de plus en plus abstraites (Piaget, 1981), qu'une base de savoir et de savoir-faire génériques se réorganisent nécessairement et constamment. Ce processus peut durer tout au long de la vie de l'individu à condition que l'individu continue à se développer, qu'il prenne soin de maintenir la neuroplasticité de son cerveau, par exemple en entreprenant de nouvelles activités, en accroissant la synchronisation du travail des hémisphères cérébraux (Chopra \& Tanzi, 2012). 


\section{LA TRANSVERSALITÉ DE LA COMPÉTENCE NARRATIVE ET SON RÔLE DANS LA GESTION DE L'INTEGRITÉ DES «SOI»}

Dans le CECRL, la narration est présente dans les descripteurs de différentes catégories d'activités du niveau A1 au niveau C1 (CECRL, 2001, p. 159). Son introduction dans le processus d'enseignement/apprentissage est précédée par le développement des capacités à décrire. Pour ce qui est de la capacité à comprendre/raconter des histoires d'un utilisateur expérimenté, celui-ci est censé savoir :

- « faire une description ou une narration élaborée, en y intégrant des thèmes secondaires, en développant certains points et en terminant par une conclusion appropriée » (CECRL, 2001, p. 49) (à l'oral en monologue suivi);

- " écrire des histoires ou des récits d'expérience captivants, de manière limpide et fluide et dans un style approprié au genre adopté » (CECRL, 2001, p. 52) (à l'écrit en écriture créative);

- « écrire des textes descriptifs et de fiction clairs, détaillés, bien construits dans un style sûr, personnel et naturel approprié au lecteur visé » (CECRL, 2001, p. 52) (à l'écrit en écriture créative).

Le CECRL envisage donc la narration comme une forme de communication interpersonnelle et comme produit de cette communication. La compétence narrative en langue seconde permet à l'utilisateur expérimenté de prendre une part active à la communication quotidienne, d'entrer en contact et d'établir des relations ainsi que de jouer divers rôles sociaux. Pourtant, les enjeux du développement de la compétence narrative en langue seconde sont encore plus importants.

Le schéma narratif en tant que représentation mentale de la structure typique du discours narratif se manifeste à travers les textes narratifs composés de $n$ séquences narratives et descriptives. Les textes narratifs s'adaptent aux normes et règles de divers genres textuels: littéraires, médiatiques, scientifiques, académiques, etc. L'acquisition de la compétence narrative en langue maternelle fait partie de l'acquisition de la compétence langagière et commence environ à deux ans et demi par une étape de co-production des récits avec un adulte (Blachowska-Szmigiel, 2010, p. 198). Les enjeux de l'organisation spatio-temporelle propre au texte narratif sont double :

- elle organise le fonctionnement de l'individu dans le monde réel, comme le constate A. Dizerbo, «C'est donc en s'appuyant sur des modèles narratifs que l'homme donne forme à ses expériences et qu'il trouve les ressources d'intelligibilité qui lui permettent d'interpréter et de donner sens à ce qu'il vit » (Dizerbo, 2014, p. 4) ;

- elle représente une structuration de base qui sous-tend l'intégrité des «SOI » (soit des images de soi-même ou des self-images). 
Le « SOI » entendu comme " une structure cognitive complexe et dynamique » (Suszek, 2007, p. 7) ${ }^{2}$ occupe une place centrale dans la personnalité et joue le rôle principal dans la régulation de divers processus mentaux, cognitifs et émotionnels,

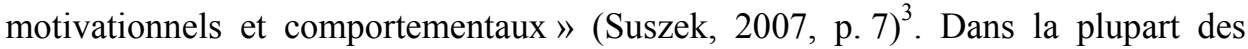
taxonomies du « SOI », la distinction s'opère à partir des critères :

- spatial (par exemple : la distinction entre le «SOI » privé, public et collectif [Triandis, 1989]),

- fonctionnel (par exemple : le « SOI » défensif [Zaborowski, 1994]),

- temporel et axiologique (par exemple : le « SOI » réel, «SOI » idéal, «SOI » imposé [Higgins, 1987]).

Force est de constater que les savoir-faire à comprendre et à écrire/raconter des histoires constituent un fondement de la communication interpersonnelle dans divers domaines de l'activité de l'individu. Les gens racontent des histoires pour se présenter, attirer l'attention, séduire, négocier, résoudre des problèmes, inventer des idées, partager les idées sur le monde et sur la vie sous forme de mythes, de littérature, d'idéologie ou de religion (Trzebiński, 2002, p. 43). Leur motifs et intentions sont donc divers. Comme le remarque J. Trzebiński, « la narration est un moyen de comprendre la réalité par les individus. Cette façon de comprendre se manifeste par le fait que, dans le flux d'événements environnants, les individus voient des histoires, se souviennent du passé comme d'une série d'histoires spécifiques et imaginent le passé [...] sous forme de scénarios d'histoires possibles. Ce ne sont pas des histoires qui arrivent aux personnes dans la vie, mais c'est plutôt la structure spécifique de leur connaissance du monde qui détermine la manière narrative de voir la réalité » (Trzebiński, 2002, p. 43) .

Comme nous l'avons déjà mentionné, la narration rend possible la compréhension et l'interprétation de ses propres expériences ainsi que de celles de l'autre (Dizerbo, 2014, p. 4). Si elle concerne l'individu lui-même, elle prend la forme d'une autonarration. L'autonarration remplit des fonctions de régulation importantes à savoir :

- L'individu trouve plus facilement le sens des événements vécus dans la vie réelle ainsi que le sens de son rôle en tant que protagonistes de ces événements. De plus, il définit plus facilement son identité. Par conséquent, il éprouve une plus grande satisfaction et a un meilleur état d'esprit dans une sphère de vie donnée.

- Les catégories de but, de moyen d'atteindre le but, d'obstacle, de lieu et de temps identifiées dans l'autonarration ont leur genèse dans l'expérience personnelle de l'individu, c'est pourquoi aident ce dernier à organiser mieux

\footnotetext{
${ }^{2}$ Traduction propre.

${ }^{3}$ Traduction propre.

${ }^{4}$ Traduction propre.
} 
ses conduites dans la vie réelle et à se concentrer plus facilement sur le but, les façons et les causes de ses comportements.

- L'individu organise mieux sa vie émotionnelle, par exemple il gère plus facilement les problèmes (Trzebiński, 2002, pp. 58-61).

Étant donnée la nécessité de jouer divers rôles sociaux majorée encore par la mondialisation du marché du travail, la gestion de l'intégrité des « SOI» ou des selfimages parait l'une des questions les plus importantes. Pourtant, comme nous l'avons déjà remarqué dans la section précédente, le CECRL la postule seulement sans l'approfondir. En remplissant divers rôles sociaux, le sujet communiquant s'interprète et se réinterprète à travers ces rôles. Il participe à plusieurs histoires différentes et en tant qu'héros de ces histoires, il reçoit des informations de retour sur ses savoirs, savoir-faire, traits de caractère, attrait physique, efficacité, etc. Les succès et les échecs dans les différents domaines de sa vie structurent la collection de ses self-images. C'est ainsi qu'à force de vivre diverses expériences et de les interpréter par un système des filtres personnels hérités des ancêtres (à savoir : les programmes de survie, les croyances, les attitudes, les valeurs, etc. [Schützenberger, 2004, pp. 35-54]) il se construit plusieurs images de «SOI» dont il se sert d'une façon souple en fonction d'une situation donnée.

Pour faire le point, l'activité narrative sous-tend : la communication quotidienne de l'individu dans différentes situations privées/professionnelles, la compréhension/l'interprétation des expériences des autres et l'intégration de l'individu dans la société. Quant à l'autonarration, elle organise et régule les conduites du sujet communicant, améliore la qualité de sa vie émotionnelle et, ce qui nous intéresse le plus dans le contexte de nos réflexions, elle favorise l'intégrité « des SOI » du sujet communicant.

\section{LE DÉVELOPPEMENT DE LA COMPÉTENCE NARRATIVE EN PHILOLOGIE ROMANE : CYCLE DE LICENCE}

Les étudiants qui arrivent en philologie romane à l'université Adam Mickiewicz représentent les niveaux hétérogènes de la compétence langagière globale en français. En Ière année du cycle de licence, nous avons des groupes de débutants, des utilisateurs élémentaires (A1, A2) et des utilisateurs indépendant du niveau B1. Il est à souligner que ces derniers sont depuis quelques années en minorité. À la fin de la IIème année du cycle de licence, les étudiants atteignent le niveau B2 et ils terminent le cycle de licence avec le niveau $\mathrm{C} 1$ certifié par l'examen final. En langue maternelle, leur compétence narrative se situe au niveau d'un utilisateur expérimenté, tandis qu'en français elle est bloquée en particulier par le niveau de compétences lexicale et morpho-syntaxique. Ce qui facilite le développement de la compétence narrative en FLE, c'est l'isomorphie du schéma formel de la narration 
en français et en polonais. Durant le cycle de licence, le statut de la compétence narrative en FLE évolue d'un outil de développement de la compétence linguistique vers un outil de communication interpersonnelle dans la vie sociale, professionnelle et académique. L'étudiant de la IIIème année puise dans sa compétence narrative pour écrire des lettres de motivation, participer aux discussions sur les sujets d'actualité et sur les phénomènes socio-culturels, rapporter le contenu des romans ou des films, développer la pensée créative, résoudre des problèmes, exprimer son avis, participer aux projets proposés dans le cadre des travaux dirigés, etc.

En ce qui concerne la place et les fonctions de l'autonarration dans le module des travaux dirigés, elle est considérée avant tout comme un outil d'expression personnelle, l'étudiant y recourt pour se présenter, raconter ses expériences, parler de son parcours universitaire et de ses projets. Abstraction faite des réflexions sur la langue et sur les stratégies d'apprentissage de la langue seconde, l'autonarration n'est pas explicitement exercée comme déclencheur d'une réflexion approfondie sur soi-même ou comme un moyen de gérer l'intégrité des «SOI». Pour attirer l'attention des étudiants sur cette fonction de l'autonarration et les sensibiliser sur les rapports entre «qui je suis » (le SOI réel) et « qui je voudrais être » (le SOI idéal), nous proposons aux étudiants de la IIème année une activité inspirée librement du test des arbres blob élaboré par P. Wilson (2009). L'objectif original de ce test est de comprendre les émotions que les individus et les groupes peuvent ressentir. Le test représente un arbre et différents personnages exerçant différentes activités. Les uns grimpent, les autres sont assis, d'autres encore traînent autour de l'arbre (voir figure 1).

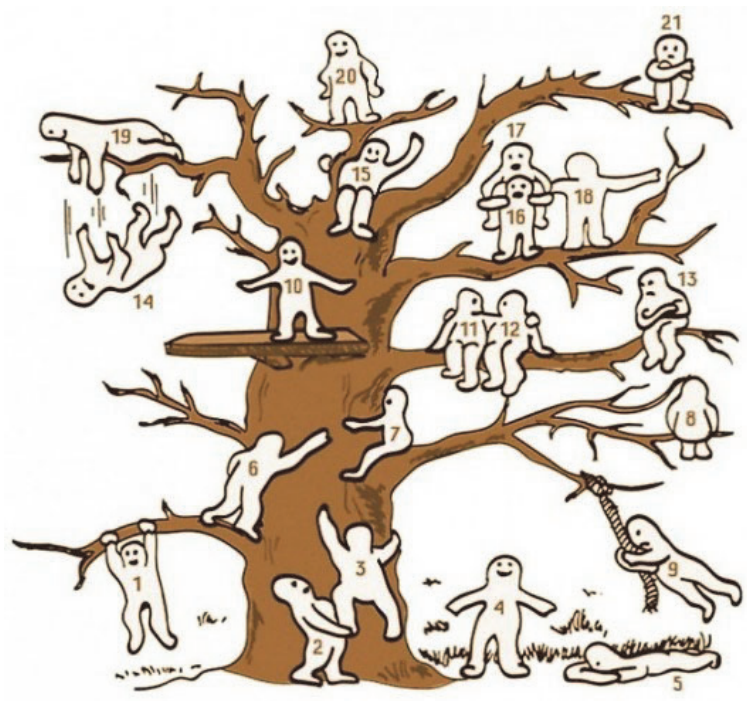

Figure 1. Arbre blob (Wilson \& Long, 2009) 
Chaque étudiant reçoit l'image en question avec un questionnaire qui suit :

Imaginez que les personnages présentés sur l'image représentent votre groupe. Chaque personnage occupe une place différente et exerce une activité différente.

1. Quelle est votre place dans le groupe? Avec quel personnage vous identifiezvous et pourquoi ? Justifiez en quelques phrases votre choix. Coloriez le personnage choisi en vert.

2. Quelles sont vos craintes (par rapport au cours, à vous-même, etc.) ?

3. Qu'est-ce que vous attendez de vous-même?

4. Qu'est-ce que vous attendez de ce cours?

5. Quelle place aimeriez-vous occuper dans votre groupe à la fin du trimestre? Serait-ce la même place ou bien une autre? Si vous pensez à une autre place, choisissez le personnage respectif, coloriez-le en rouge et justifiez-votre choix en quelques phrases. Si votre place reste la même, justifiez ce choix en quelques phrases.

L'activité en question est effectuée au début du troisième trimestre en atelier de créativité (l'un des cours dans le module des travaux dirigés) et répétée à la fin du trimestre. Cette fois-ci, les étudiants reçoivent d'abord un nouveau test à remplir avec les questions suivantes :

1. Quelle est votre place dans le groupe actuellement?

2. Avec quel personnage vous identifiez-vous et pourquoi? Justifiez en quelques phrases votre choix. Coloriez le personnage choisi en jaune.

3. Est-ce que ce cours a répondu à vos besoins?

Après avoir fait l'activité, les étudiants reçoivent leur test du début du trimestre. Ils ont à comparer leurs réponses et à formuler leurs conclusions sur l'évolution de leur place dans le groupe. Au début du trimestre, ils peuvent choisir entre le français et le polonais pour répondre au test ; à la fin du trimestre, il leur est demandé de rédiger leurs réponses en français. Les résultats de cette comparaison sont discutés en groupe en français, les étudiants répondent à l'oral aux questions suivantes :

1. Qu'est-ce qui vous a surpris?

2. Dans quelle mesure avez-vous atteint la place espérée?

3. Êtes-vous satisfait de la direction de votre évolution?

En voici quelques exemples des réponses d'une étudiante. Nous les citons en gardant l'orthographe et la grammaire originales. Au début du trimestre, elle s'est identifiée avec le personnage $n^{0} 6$ et elle aimerait être le personnage $n^{0} 15$. Elle a justifié son choix en polonais de façon suivante : «Widzę siebie w tym miejscu, ponieważ dopiero od niedawna zaczęłam naukę francuskiego. Jestem jak ta postać i dopiero się wspinam. Mimo to kiedy potrafię, udzielę pomocy innym. Wszyscy jesteśmy tu, aby czegoś się nauczyć. Jestem nauczona, że ciężka praca zawsze wychodzi nam na dobre i prowadzi na szczyt $»^{5}$. À la fin du trimestre, l'étudiante

\footnotetext{
${ }^{5}$ « Je me vois ici car je viens de commencer à apprendre le français. Je suis comme ce personnage, je grimpe. Cependant, quand je peux, j'aide les autres. Nous sommes tous ici pour apprendre quelque
} 
s'est identifiée avec le personnage $\mathrm{n}^{\circ} 10:$ «Je ne suis pas encore là où j'aimerais être mais je suis sur la bonne voie et je suis très contante de ça ». Quant à l'évaluation du cours, elle a constaté : "Je pense que ce cours m'a permis de s'ouvrir au monde». En ce qui concerne les réponses à d'autres questions du questionnaire du début du trimestre, je les présente dans le tableau ci-dessous.

\begin{tabular}{|l|l|}
\hline \multicolumn{1}{|c|}{ Questions } & \multicolumn{1}{|c|}{ Réponses } \\
\hline $\begin{array}{l}\text { 6. Quelles sont vos craintes (par rapport au cours, } \\
\text { à vous-même, etc.)? }\end{array}$ & $\begin{array}{l}\text { Obawiam się, że nie dorównam innym, którzy } \\
\text { mają więcej umiejętności i wiedzy. Obawiam się } \\
\text { także braku akceptacji }\end{array}$ \\
\hline 7. Qu'est-ce que vous attendez de vous-même? & Motywacji, mobilizacji, wytrwałości ${ }^{7}$. \\
\hline 8. Qu'est-ce que vous attendez de ce cours? & $\begin{array}{l}\text { Chcę polepszyć swoją znajomość języka fran- } \\
\text { cuskiego. Tworzyć ciekawsze teksty }\end{array}$ \\
\hline
\end{tabular}

Figure 2. Tableau regroupant les réponses aux questions 6, 7, 8, du questionnaire

L'étudiante dont les réponses ont été citées ci-dessous, se montre comme quelqu'un de sérieux qui a l'ambition d'améliorer son niveau de français, de la motivation et une attitude positive. Pourtant ce qui ressort de ses réponses c'est l'anxiété langagière : elle se compare aux autres membres du groupe et craint de ne pas être acceptée car son niveau est moins élevé que celui des autres. Elle a besoin des renforcements positifs au sein du groupe pour se sentir plus à l'aise.

Les réponses des étudiants sont une source d'informations sur l'évolution de leur compétence langagière, de la dynamique du groupe, des rapports interpersonnels, de leurs besoins individuels et de leurs anxiétés ainsi que de leurs représentations de l'activité d'apprentissage. L'enseignant peut en profiter pour organiser/réguler le processus d'enseignement. Aux étudiants, cette activité donne la possibilité de réfléchir sur leurs besoins et attentes, de s'autoévaluer, de prendre conscience de « qui je suis » et « de qui j'aimerais être » et de formuler les objectifs à court terme. Ce qui constitue les limites de cette activité c'est en premier lieu le niveau de langue. Pour les étudiants de la IIème année, le français n'est pas une langue d'autonarration ni au début, ni à la fois du troisième trimestre : pendant la

chose. On m'a appris que le travail dur était toujours bénéfique et nous conduit au sommet » (traduction propre).

${ }^{6}$ « Je crains de ne pas rattraper les autres qui ont plus de compétences et de connaissances. J'ai aussi peur du manque d'acceptation » (traduction propre).

${ }^{7}$ « Motivation, mobilisation, persévérance» (traduction propre).

${ }^{8}$ « Je veux améliorer ma connaissance du français. Créer des textes plus intéressants » (traduction propre). 
discussion des résultats du test ils avaient besoin de recourir aussi au polonais. À part le niveau de langue, l'enseignant doit tenir compte du caractère tendancieux des réponses. Pour minimiser ce risque, il convient de précéder le test par une explication de ses objectifs et par une discussion de ses enjeux.

\section{CONCLUSION}

Comme nous l'avons souligné, le sujet communicant doit savoir établir les relations sociales et jouer divers rôles sociaux dans le contexte plurilingue et pluriculturel. Pour ce faire, il a besoin de développer les compétences transversales parmi lesquelles se trouve aussi la compétence narrative. Constituant un fondement de la communication quotidienne, elle permet au sujet apprenant de remplir son rôle d'acteur social et de gérer l'intégrité des self-images. Cette dernière fonction de la compétence narrative est peu explorée dans le contexte de l'enseignement/apprentissage du FLE en philologie romane. Pourtant, étant donné le postulat du CECRL concernant le développement harmonieux de la personnalité et de l'identité, il serait souhaitable de montrer aux étudiants l'utilité de la compétence narrative pour la gestion des «SOI». L'activité inspirée du test des arbres blob donne une telle possibilité à condition qu'elle soit effectuée régulièrement et suivi par une discussion des résultats. La question se pose alors de savoir dans quelle mesure et comment nous pourrions montrer aux étudiants d'autres outils de gestion des « SOI » dans l'objectif de développer leur conscience et de leur faciliter l'accroissement de leur « pouvoir personnel» (ang. empowerment). 


\section{BIBLIOGRAPHIE}

Bertelle, L. (2011). Dwujęzyczność w świetle najnowszych badań naukowych. Lingwistyka Stosowana, 4, 241-243. Retrieved from https://portal.uw.edu.pl/documents/7276721/../LS4_ 2011_art_BERTELLE.pdf.

Blachowska-Szmigiel, M. (2010). Twórcze schematy poznawcze a kreatywność językowa. Na przyktadzie języka francuskiego jako obcego. Poznań : Wydawnictwo Naukowe UAM.

Chopra, D., \& Tanzi, R.E. (2012). Twój supermózg. Warszawa : Wydawnictwo Olé.

Coste, D. et al. (2001). Un cadre européen commun de référence pour les langues - apprendre, enseigner, évaluer. Paris : Conseil de l'Europe / Les Éditions Didier.

Coulet, J.C. (2016). Compétence, compétences transversales et compétences clés : peut-on sortir de l'impasse ? Éducation et Socialisation [En ligne], 41, mis en ligne le 19 juillet 2016. Retrieved from http://journals.openedition.org/edso/1708. DOI : 10.4000/edso.1708.

Dizerbo, A. (2014). Apprendre à exister : enjeux et modalités d'une éducation narrative. Le sujet dans la Cité 2, 5, 161-171, mis en ligne le 30 décembre 2014. Retrieved from http://www. cairn.info/revue-le-sujet-dans-la-cite-2014-2-page-161.htm.

Higgins, E.T. (1987). Self-discrepancy. A theory relating self and affect. Psychological Review, 94, 319-340.

Piaget, J. (1981). Równoważenie struktur umysłowych. Centralny problem rozwoju. Warszawa : Państwowe Wydawnictwo Naukowe.

Schützenberger, A.A. (2004). Secrets, secrets de Famille et Transmissions Invisibles. Cahiers Critiques de Thérapie Familiale et de Pratiques de Réseaux 2, 33, 35-54, mis en ligne le 1 octobre 2005. Retrieved from https://www.cairn.info/revue-cahiers-critiques-de-therapiefami liale-2004-2-page-35.htm. DOI : org/10.3917/ctf.033.0035.

Suszek, H. (2007). Różnorodność ja. Roczniki Psychologiczne, t. X, nr 2, 7-37.

Triandis, H.C. (1989). The self and social behavior in differing cultural contexts. Psychological Review, 96, 506-520.

Trzebiński, J. (2002). Narracja jako sposób rozumienia świata. Gdańsk : Gdańskie Wydawnictwo Psychologiczne.

Wilson, P., \& Long, I. (2009). The Big Book of Blob Trees. London : Speechmark Publishing Ltd. www.lemonde.fr/emploi/article/2016/10/12/en-entreprise-la-personnalite-des-diplomes-devientpri mordiale 5012299 1698637.html

Zaborowski, Z. (1994). Wspótczesne problemy psychologii społecznej i psychologii osobowości. Warszawa : Profi. 